
temps de la convergence?

\title{
Réseaux virtuels, reconstruction du lien social et de l'identité dans la diaspora noire
}

Virtual Networks, the Reconstruction of Social Ties, and Identity in the African and Black Diaspora

\section{Alain Kiyindou et Théodora Miéré Pélage}

\section{OpenEdition} Journals

Édition électronique

URL : http://journals.openedition.org/edc/3425

DOI : $10.4000 /$ edc. 3425

ISSN : 2101-0366

Éditeur

Université Lille-3

Édition imprimée

Date de publication : 30 juin 2012

Pagination : 189-201

ISBN : 978-2-917562-07-9

ISSN : $1270-6841$

Référence électronique

Alain Kiyindou et Théodora Miéré Pélage, « Réseaux virtuels, reconstruction du lien social et de l'identité dans la diaspora noire », Études de communication [En ligne], 38 | 2012, mis en ligne le 30 juin 2014, consulté le 21 avril 2019. URL : http://journals.openedition.org/edc/3425 ; DOI : 10.4000/ edc.3425

Ce document a été généré automatiquement le 21 avril 2019

(c) Tous droits réservés 


\section{Réseaux virtuels, reconstruction $d u$ lien social et de l'identité dans la diaspora noire}

Virtual Networks, the Reconstruction of Social Ties, and Identity in the African and Black Diaspora

Alain Kiyindou et Théodora Miéré Pélage

1 La cybersocialité ou lien social par les TIC est actuellement en débat avec d'un côté les tenants d'une société virtuelle, vue comme un nouveau réel qui transcende le monde et capable de se substituer à lui et de l'autre, ceux qui s'inquiètent des conséquences des rapports basés sur des relations virtuelles et qui évoquent des risques (risque de violence, risque de tautisme, etc.). La diaspora africaine s'est particulièrement illustrée dans l'usage des dispositifs techniques afin de recréer voire de maintenir le lien avec cette partie d'elle restée sur le continent d'origine. Il s'agit, ici, d'une population issue de la déportation africaine à l'époque de la traite esclavagiste $\mathrm{duXVI}^{\mathrm{e}}$ au XIX ${ }^{\mathrm{e}}$ siècle, des migrants et de leurs descendants. Pour les autorités africaines, la diaspora est la sixième sous-région du continent noir. Pour les institutions internationales, elle est le socle des politiques de co-développement. En effet, les transferts financiers des émigrés vers leur pays d'origine représentent une manne en constante évolution. Dans certains États, ils dépassent l'aide au développement et contribuent de manière décisive au revenu national (afriqueavenir.org/2011). À côté de cette contribution financière et économique, se développent des échanges plus intellectuels, notamment grâce aux TIC. Il devient donc intéressant de voir comment, à travers des dispositifs techniques, les Africains de la diaspora essaient de reconstruire le lien rompu avec la communauté d'origine et comment les TIC répondent aux préoccupations de reconstruction du lien social.

2 Pour réaliser cette étude, nous avons procédé à une analyse des sites web portant sur la diaspora africaine qui affichent dans leurs balises le mot clé «diaspora africaine». Ce choix peut paraitre restrictif, mais il a l'avantage de réduire de façon pratique le champ 
de la recherche. En nous intéressant principalement aux dix premiers sites affichés, notre analyse s'en est trouvée facilitée. Parmi les sites se déclarant panafricanistes se trouvent : congopages.com, afrikadiaspora.com, afrocom.org, grioo.com, mwinda.org, afriblog.com, afriquetandem.com, mukanda.org, pratic (joretic.com), afrik.com. Ils se distinguent par leurs contenus, leurs noms, le choix des couleurs et des visuels.

Ce travail s'inscrit dans le cadre de la communication pour le développement telle que définie par le professeur Nora Québral, c'est-à-dire l'usage des moyens de communication, la transmission de nouvelles connaissances en vue de l'amélioration des conditions de vie et du bien-être des populations (Bessette, 2004). En effet, on est passé d'une approche descriptive de la diaspora comme ensemble des membres d'une communauté dispersée à des définitions plus fonctionnalistes comme celle de Pires Hester qui considère la diaspora comme «l'utilisation stratégique de l'identification ethnique à une patrie d'origine pour lui faire bénéficier des systèmes et des institutions de la patrie d'accueil » (Pires-Hester, 1999, 486). Il y a donc dans la première perception une recherche d'amélioration des conditions de vie du pays ou de la région d'origine et, dans ce cadre, les TIC apparaissent comme le moyen le plus sûr de dynamiser les actions de développement. À titre d'exemple, le Fonds des Nations Unies pour l'Afrique s'est engagé dans la création du Réseau de la Diaspora Numérique pour l'Afrique, le projet DDNA (Digital Diaspora Network for Africa) dont l'objectif est de mobiliser les compétences technologiques, managériales et professionnelles des membres de la diaspora africaine en Europe et aux États-Unis, afin d'accélérer le transfert d'informations et des Technologies de l'Information et de la Communication.

Quant au terme lien social que nous utilisons ici, puisque c'est bien de reconstruction du lien social dont il est question, nous empruntons à Farrugia sa conception selon laquelle « les valeurs constitutives du lien social sont celles qui, dans la conscience et l'imaginaire collectifs, ont du sens pour les membres d'une société donnée quant à la co-gestion de ce qu'ils estiment être un bien commun à préserver dans l'intérêt général de la société concernée » (Farrugia, 1993, 40). Le lien social apparait donc ici comme ce qui maintient, entretient une solidarité entre les membres d'une même communauté, c'est-à-dire ce qui permet la vie en commun.

\section{La construction du lien social}

5 Plusieurs pays d'Afrique noire se caractérisent par l'existence de réseaux complexes de solidarités sociales et affectives : on se visite, on se soutient dans le bonheur (naissance, mariage, baptême, etc.) comme dans le malheur (hospitalisation, décès, etc.). C'est dans ce contexte que se vit une vie sociale très riche. En France, depuis une dizaine d'années, d'importants phénomènes de changement sont clairement observables notamment sur Afrikadiaspora et Africom. Il y a la revendication d'une identité collective noire qui ne cesse d'augmenter, une identité culturelle et des spécificités. Au-delà de ces revendications, on assiste en France et ailleurs à la création de nouveaux types et lieux de commerce où les membres de la diaspora se regroupent. Claire Scopsi (2004) a analysé les téléboutiques de Château Rouge dans le dix-huitième arrondissement de Paris où on assiste à une inversion du transfert de modèle. Mais si l'accès aux Technologies de l'information et de la communication parait facile en France, de nombreuses difficultés demeurent dans les pays d'origine. Elles sont liées à ce qu'on appelle communément la fracture numérique. 
6 Recréer le lien social ou lutter contre la rupture sociale par l'utilisation des moyens de communication n'est pas chose nouvelle. Dans le passé, les diasporas issues de l'esclavage se sont appuyées sur les moyens de communication à leur disposition pour lutter contre cet isolement. Ils ont pu, à travers les chants, les danses, les contes, reconstituer le lien social et perpétuer la mémoire. Mais le lien avec la famille, les membres de la communauté d'origine s'est surtout fait avec le courrier, puis le téléphone qui a permis aux membres de l'étranger de participer à la vie du groupe. En Afrique noire, les expatriés ont joué un rôle important dans le développement des usages des TIC (Miere-Pélage, 2008). En effet, ils ont acquis pour leur famille « restée au pays » des téléphones mobiles qui, une fois débloqués, pouvaient être utilisés sur place. La possibilité offerte par le téléphone mobile d'avoir un lien permanent et la baisse des prix des terminaux dans les pays développés ont été des facteurs très importants dans l'équipement des africains du continent.

7 Internet - et plus spécifiquement le web 2.0 - a ceci d'intéressant qu'il facilite les retrouvailles entre des amis dont on a perdu la trace. C'est comme si la technologie se mettait soudain à recoller les fragments de la vie sociale, à rompre l'isolement, à souder les fractures. Facebook est résolument l'application qui favorise le mieux les liens sociaux notamment à travers ses dispositifs de recherche d'amis potentiels. La révolution essentielle opérée par le web 2.0 résulte principalement dans le fait qu'il permet à l'internaute de tisser sa toile, d'étendre son réseau et de se croire le principal acteur de son espace social. Si les possibilités offertes par le web 2.0 d'accroître les réseaux sociaux ne peuvent être négligées, celui-ci n'a pas l'exclusivité du lien social. On connaît également l'engouement des Africains à faire vivre les cérémonies de mariages, les obsèques, les fêtes de retrait de deuil à travers l'échange des cassettes vidéo. Le site Internet Mwinda (www.mwinda.org) diffuse systématiquement des communiqués nécrologiques ainsi que les informations relatives aux veillées mortuaires. Aujourd'hui, des vagues plus récentes se trouvent confrontées à la fois à la recherche de la continuité par rapport à l'expérience pré-migratoire et à la nécessité de s'adapter aux cadres économiques, sociaux et politiques du pays d'accueil. Internet apparaît donc comme un moyen efficace dans la reconstruction du lien social et le cyberespace fait partie des territoires dans lequel les Africains se retrouvent réellement. Un espace au sein duquel on peut critiquer les actions des gouvernements en place dans les pays d'origine, sans risque d'être censuré ou de voire sa vie menacée (mwinda.org...). Un territoire sur lequel on peut circuler, avec ses repères, à côté des personnes qui partagent les mêmes valeurs culturelles (afrik.com). Les sujets qui reviennent le plus souvent sur les sites que nous avons étudiés sont : les actualités des pays africains, les traditions africaines, l'histoire (esclavage, colonisation, etc.), la constitution d'une conscience collective, la prise de conscience des membres de la diaspora des problèmes qui les concernent, la négritude, le panafricanisme, l'actualité de la diaspora. Sur ces sites, les sujets d'actualité du pays d'accueil côtoient ceux du pays d'origine. Ils se présentent généralement sous la forme d'un texte illustré, signé par son auteur, sur lequel les internautes sont invités à échanger. Elle est comprise à travers l'histoire du monde, celle du continent africain et celle de chaque conflit. Les sites les plus prisés sont ceux qui traitent des informations générales et les sites des associations. Ce qui explique leur développement, c'est la vulgarisation de la création des blogs (http://kouamouo.ivoire-blog.com/, http:// obambegakosso.unblog.fr) qui se font aujourd'hui en quelques minutes avec des outils clé en main sans avoir des notions de programmeur. Internet reste un moyen d'expression et 
d'existence pour la diaspora qui véhicule ses messages et ses activités. Afrikadiaspora (http://www.afrikadiaspora.com) est sans conteste un de ceux qui représente le mieux ce que pourrait être l'Internet de la diaspora africaine. Ce site panafricaniste rappelle, de par ses couleurs, la terre, terre des ancêtres. On peut d'ailleurs voir sur la manchette la silhouette d'un sage à côté d'un arbre qui pourrait fort bien être un arbre à palabre. Sur son blog, Alain Mabanckou, écrivain d'origine congolaise écrit une phrase selon laquelle la littérature gabonaise se résumerait à quelque quatre textes. Cette déclaration soulève une réflexion populaire sur ce qu'est la littérature et sur l'état de la littérature gabonaise. Cette affirmation publiée sur le blog de l'auteur a engendré plus de 200 pages de commentaires et entraîné la création de sites dédiés à cette question. Au Gabon, le débat sur Internet a été repris par les étudiants de littérature africaine qui, tous les jours, affichaient dans les locaux de la faculté des lettres, les nouvelles pages de commentaires.

\section{La reconstitution identitaire}

L'identité est un construit social dans lequel les représentations, les images, la mémoire jouent un rôle important. C'est aussi ce par quoi nous fondons notre reconnaissance ; ce qui fait qu'il y a « nous et les autres » c'est-à-dire ce qui à la fois nous lie et nous distingue des autres. En Afrique, cette identité se construit dans la relation sacrée avec les parents, donc à travers le cercle familial, le clan, le lignage. En effet, en Afrique, l'individu est, durant toute sa vie, inscrit dans un rapport de participation et d'assistance avec sa famille, son lignage et sa communauté. À côté des liens sociaux fondés sur la parenté biologique se développent d'autres formes de sociabilité non moins importantes dans la définition des rapports sociaux. Ces nouvelles formes de parenté sont scellées par des transactions et conventions sociales telles que l'alliance, le pacte, le serment, la confraternité, l'amitié. Ces liens prennent corps à travers des espaces sociaux et institutions comme les classes d'âge, les cercles initiatiques, les mutuelles de travail.

Toutes ces relations sont très importantes pour la reconnaissance identitaire des Africains, mais pour beaucoup, ces relations ont été rompues avec l'éloignement physique: les morts ne sont plus honorés, les cercles, les mutuelles ne sont plus fréquentées, ce qui renforce le déséquilibre identitaire. Les TIC participent donc à la reconstruction de cette identité, en ce qu'elles permettent un contact, ne fut-ce que virtuel avec les amis, les membres de la famille restés «au pays». Avec les forums électroniques et le développement des " chat " vocaux, on voit s'organiser des réunions de famille via Internet où chacun, après les salutations d'usage, prend la parole tout en respectant toutes les précautions en vigueur. Mais un usage assez particulier des TIC réside dans l'utilisation d'Internet pour la mémoire des disparus. On voit aujourd'hui fleurir, sur l'Internet africain, des « necronet ", sorte de cimetière virtuel, permettant aux membres de la famille dispersés dans le monde entier de renouer le lien rompu avec les ancêtres, les morts. Il s'agit donc d'une traduction matérielle d'une vision abstraite des ancêtres, traduction qui prend appui sur la réalité solide des technologies et donne une illusion de réalité. On a, sur Afrocom, l'impression de se retrouver devant la tombe d'un de ces ancêtres, et ces sites sont abordés avec le même respect que dans la réalité. Le necronet est donc la réponse technique à l'impossibilité constatée de maintenir le lien avec les morts. Ce contact permanent est d'autant plus important que pour les Africains les morts ne sont pas morts. 


\section{L'émergence des « territoires »}

10 Les éléments caractéristiques d'un territoire sont les relations d'occupation et de dominance qu'un individu ou un groupe entretient vis-à-vis d'un lieu. Vu sous cet angle, Internet est donc un territoire, puisqu'il est, pour reprendre les termes d'Olivier Zablocki, " un peu notre espace de vie sociale, mentale, intellectuelle, artistique, économique " (Zablocki, 2001, 35), un lieu que jeunes et moins jeunes, hommes et femmes, quelle que soit leur origine, investissent. On peut retrouver dans les sites que nous avons étudiés les trois types de territorialité que distinguent Altman et Chemers (Altman, 1984) à savoir le territoire primaire, secondaire, tertiaire.

11 Le territoire primaire, lieu "privé ", clairement identifié comme appartenant à un sujet particulier est le lieu de l'« intimité » par excellence. C'est sur ce territoire que se situent les nombreuses pages personnelles, espaces d'expression individuelle qui permettent de se mettre en scène, de faire partager ses passions, ses idées, etc. Mais en dehors de ce caractère ouvert, le territoire primaire est le lieu du sacré mais aussi du secret, ces choses que l'on partage en famille et qui fortifient et confortent la solidarité. En effet, de nombreux sites sur l'Afrique permettent de partager des souvenirs, des récits de voyage, des images pittoresques du continent. Comme pour mieux instaurer le dialogue, leurs concepteurs n'hésitent pas à se présenter à travers une page « qui suis-je » ou « tout sur moi », véritable carte d'identité de l'auteur avec photo, état civil, passions. Il y a sur ce territoire des sites personnels où les auteurs se présentent, mettent leurs photos de famille et parfois même leur journal intime. Une des pratiques constatées est l'utilisation de mots de passe assez simples : on utilise un code qui permet de filtrer les accès. Très souvent le mot de passe est le nom de la page sur lequel se trouve la photo. Cette pratique est courante lors d'une naissance dans la famille: au lieu d'envoyer la photo à toute la famille dispersée, on crée un site et en invite les membres de la communauté à s'y retrouver. Avec la vulgarisation et la banalisation des logiciels de création de site, apparaît un nouveau genre, des sites collaboratifs créés par des membres dispersés de la famille. Il s'agit principalement de galeries de photos rappelant les événements marquants de la famille. Les quelques spécialistes en la matière sont sollicités par les amis, les cousins, etc. Les mêmes pratiques existent en France et en Europe, mais pour les immigrés, cela donne une signification particulière...

Le territoire secondaire est un lieu associé à une unité sociale intermédiaire. Les regroupements observés sur ce territoire se fondent presque toujours sur la recherche du même. Ce même peut être un pays, une langue, un village, une région, une appartenance ethnique. C'est là que seront traités les problèmes de développement du village, du quartier, des opérations de charité pour aider tel ou tel autre membre, des opérations humanitaires pour construire des dispensaires, des écoles, etc. On peut constater sur les sites un certain nombre de rituels, des signes de reconnaissance spécifiques qui n'ont d'autre but que de fortifier le petit groupe. Cette communauté a également des principes, des symboles, des références. Faire partie de la communauté, c'est donc partager ces points communs. Ces complicités se bâtissent à travers les échanges sur des forums et des " chat ». C'est là que les membres du groupe viennent se libérer du poids de la parole.

Les groupes les plus visibles sur les TIC sont les associations familiales, les associations des originaires d'une même ville ou d'un même village, les associations ethniques, les associations d'anciens élèves, les associations religieuses. C'est bien souvent à travers ces 
organisations que les Africains de la diaspora entretiennent des liens avec leur pays d'origine. S'ils ont des compétences professionnelles et une expérience, ils les mettront généralement au service de ces organisations. Par exemple, La communauté Afrique Tandem (http://www.afriquetandem.com) organise en France, des collectes de livres et manuels scolaires au profit des établissements scolaires de Bamako et de Mopti au Mali. L'association PRATIC (Promotion, Réflexion, Analyse sur les Technologies de l'Information et de la Communication) se fixe comme objectif d'œuvrer pour le développement par le biais des TIC (Technologies de l'Information et de la Communication) au Congo-Brazzaville. Elle dispose d'un site Internet sur lequel elle informe le public de ses actions et de ses projets, notamment des JORETIC, journées de réflexion sur les TIC organisées à Paris et à Brazzaville.

Le territoire tertiaire ou "public », bien qu'accessible à tous, rappelle également son attachement au continent africain à travers un certain nombre de marques. En effet, on trouve sur les sites de ces communautés un ensemble d'éléments iconiques parmi lesquels le masque, la carte de l'Afrique, la case, l'arbre à palabre, mais on y trouve également des proverbes africains, des contes, des citations de personnages historiques etc. C'est ici qu'on classera les sites des ONG de développement, des groupes politiques... Internet propose une diversification de l'offre médiatique, une autre image de l'Afrique, de ce qui fait son actualité et son histoire. Le public qui fréquente ce territoire cherche des informations sur les investissements en Afrique, c'est-à-dire les différentes expériences menées. Il cherche également des pistes pour les aider à réaliser leurs projets, et les témoignages sont très importants ici.

Le territoire tertiaire est généralement ouvert au «hors groupe " mais les membres savent en limiter l'accès. Les modérateurs du site des journalistes africains en exil JAFE (www.afriblog.com), par exemple, chassent impitoyablement tous les non-membres de l'association. Ce qui paraît paradoxal, puisqu'il s'agit du site de la communauté des journalistes menacés par des régimes autocratiques donc connaissant la censure et l'exclusion. On constate donc, dans ces communautés, la présence d'un « noyau dur » qui, en principe, accueille et s'efforce d'intégrer les nouveaux, mais qui joue surtout un rôle de garde-fou.

16 La diaspora participe au développement du continent et les gouvernements africains commencent à mesurer la portée de sa contribution. Prenant la parole à l'occasion d'ADF 2000, le président Musevini a décrit les Ougandais de l'extérieur comme étant la ressource la plus importante du pays. Les Ougandais de la diaspora envoient en effet en Ouganda quelque 400 millions de dollars chaque année, montant qui est supérieur aux recettes d'exportation du café, le premier produit agricole de ce pays (source: afriqueavenir.org/2011). C'est dans ce contexte que le gouvernement béninois a créé un site destiné à la diaspora béninoise. Il permet de fédérer les contributions au développement des professionnels et investisseurs béninois de l'étranger. Un certain nombre de contacts officiels permettent de leur faciliter les démarches. Ils peuvent également s'inspirer des témoignages de leurs compatriotes. À côté de ces entreprises sérieuses, on va trouver sur ces sites des rumeurs. Sur l'un des sites destinés à la diaspora, grioo.com, un espace a été créé après la mort du footballeur camerounais Marc Vivien Foé, avec un forum permettant au public d'exprimer sa douleur. Ce site se présente d'ailleurs comme un portail proposant des nouvelles brèves, des articles et des forums de discussion sur le continent et les communautés d'origine africaine dans le monde. 


\section{Du virtuel au présentiel}

17 Pour des personnes habituées au présentiel et fortement attachées à l'oralité, la relation par Internet montre vite ses limites avec notamment l'absence du corps auquel on va essayer de remédier à travers le recours à l'image et l'organisation des rassemblements. Le recours à l'image se fait par le biais de la visioconférence, une technologie qui malheureusement n'a pas encore atteint le succès attendu, mais qui apporte une grande satisfaction dans le cadre des relations avec les membres de la famille restés au pays. Dans le cas des personnes qui ne se connaissent pas vraiment, on va essayer dans les différentes communautés bâties sur le Web de donner un corps aux différents pseudonymes en offrant la possibilité à ceux qui le désirent de mettre leurs photos en ligne, créant ainsi de véritables galeries de portraits numérisés des usagers (ou «trombinoscope ») avec leurs adresses électroniques ou des liens hypertextes vers leurs pages personnelles. Sur Grioo.com, par exemple, un agenda vient préciser les lieux et les dates des événements jugés importants pour la communauté.

Internet ne se suffit donc pas à lui seul et les faits montrent que c'est un leurre de croire qu'une société bâtie sur des dispositifs technologiques peut fonctionner et perdurer sans d'autres modes d'existence parallèles. Comme l'a déjà observé Josiane Jouët à propos des communautés télématiques (Jouët, 1992) il y a dans ces communautés une " hybridité de modes de communication ", une transformation de la virtualité à travers ce qui, selon les contextes linguistiques et culturels, sera appelée rassemblements, conventions, ou get together. Ces réunions en face-à-face sont initiées par un membre influent du groupe, mais au-delà du statut de l'initiateur, il faut que l'idée plaise. Ces rassemblements sont souvent des moments de détente, mais on voit de plus en plus s'organiser des réunions de travail ( Pratic à travers le site joretic.com), comme si Internet n'était bon qu'à servir d'amorce, comme s'il manquait quelque chose dans le monde virtuel pour que le groupe continue à exister et réalise ses projets. Cette chose qui manque pourrait bien être le cadre institutionnel qu'exigent certaines opérations. C'est sans doute ce qui explique qu'Africagora (http://www.afrik.com/p/africagora/) organise à Paris son huitième forum annuel pour évoquer l'intégration économique et sociale des populations et décideurs originaires d'Afrique, de la Caraïbe et du Pacifique, etc.

Ce glissement du virtuel au présentiel, visible notamment sur les forums africains, est de plus en plus constaté, mais il ne faut pas oublier que le point de départ, en tout cas pour ce qui est de la diaspora, est le monde réel, le lien avec la terre d'origine. Il y a donc eu un premier glissement du monde « réel » au monde virtuel, et maintenant s'établissent des va-et-vient permanents.

\section{Conclusion}

Les TIC constituent une matrice d'où peuvent constamment jaillir de nouveaux territoires virtuels, tels des villages dont la somme forme un ensemble d'espaces habités. Dans cet ensemble, les internautes peuvent aller d'un village à l'autre. Le « zapping » de forum à forum, de site à site..., ne permet pas de s'attacher et de s'engager dans de grands projets. Il s'agirait donc plutôt de "contractualisme éphémère ", un contractualisme bref mais intense. 
21 Les TIC sont l'arbre à palabre de ceux qui du fait de leur émigration sont privés de l'arbre à palabre réel et c'est en cela qu'il est intéressant pour les personnes de la diaspora si éloignées et si proches de leurs familles, de leur pays, de leurs cultures d'origine. Mais il faut se rappeler - et c'est d'ailleurs ce qu'affirme Gérard Dubey - que « l'efficacité de ces technologies à supporter les relations dépend pour l'essentiel de l'existence, ou plutôt de la préexistence d'un cadre institutionnel, d'un cadre social » (Dubey, 2001, 40) En tous cas, il existe dans le cadre des diasporas africaines un fort attachement à leur communauté d'origine, un besoin très fort de lien social, donc un terrain très favorable sur lequel les dispositifs technologiques viennent s'appuyer. En effet, les technologies virtuelles sont impuissantes à produire à elles seules du lien social ni même les conditions nécessaires à son épanouissement. Leur efficacité constatée auprès de la diaspora africaine dépend presque entièrement de leur besoin de recherche de continuité avec leur vie prémigratoire. Mais la combinaison entre les possibilités techniques des technologies de l'information et de la communication et la ferme volonté des Africains d'œuvrer pour la visibilité de leur culture, le bien-être de leurs familles, le développement de leur village, leur quartier, de leur région, leur pays, leur continent constituent un socle puissant sur lequel peut être bâtie une communauté virtuelle.

\section{BIBLIOGRAPHIE}

Altman I. et Chemers M., (1984), Culture and environment, New York, Cambridge University Press, $337 \mathrm{p}$.

Bessette G., (2004), Communication et participation communautaire, Laval, Presses de l'Université de Laval, $138 \mathrm{p}$.

Bessette G., (2011), La communication pour le développement en Afrique de l'Ouest et du centre : vers un agenda d'intervention et de recherche, http://cassandria.wordpress.com/2010/06/24/

communication-participative-pourledeveloppement/, date de la dernière visite :

30 septembre 2011.

Birago D., (1961), Les contes d'Amadou Koumba, Dakar, Présence Africaine, 189 p.

Biyaoula D., (1997), L'Impasse, Paris, Présence Africaine, 327 p.

Laye C., (1953), L'Enfant Noir, Paris, Editions Plon, 332 p.

Cheneau-Loquay A., (2007), La connexion internationale de l'Afrique sub-saharienne, entre marché et bien public, in Mathien M., Le Sommet mondial sur la société de l'information et après, Bruxelles, Bruylant, pp. 216-238.

Cheneau-Loquay A. (dir.), (2004), Les fractures numériques Nord/Sud en question, NetSuds $n^{\circ} 2$, septembre, $450 \mathrm{p}$.

Cheneau-Loquay A. (dir.), (2004), Mondialisation et Technologies en Afrique, Paris, Karthala, pp. 275-293. 
Diminescu D., (2005), Le migrant connecté. Pour un manifeste épistémologique, Migrations/Sociétés, vol. 17, n¹02, pp. 275-292.

Dubey G., (2001), Le lien social à l'ère du virtuel, Paris, PUF, 258 p.

Farrugia F., (1993), La crise du lien social, essai de sociologie critique, Paris, L'Harmattan, 223 p.

Guignard T., (2004), Les accès publics à Internet au Sénégal : une émergence paradoxale, in Kiyindou A. (dir.), Communication et dynamiques de globalisation culturelle, Paris, L'Harmattan, 327 p.

Kiyindou A., (2004), L'Arbre à palabre domine la forêt électronique, in Hermes n40, novembre, pp. 146-149.

Kiyindou A., (2008), Société de l'information : Francophonie et culture africaine, in Fendler U. et Lusebrink H. J. (dir.), Francophonie et globalisation culturelle, Francfort, IKO, pp. 223-234.

Kiyindou A., (2005), Un continent oublié des médias occidentaux. Une Afrique sans histoire, in Mathien M. (dir.), La médiatisation de l'Histoire, ses risques et ses espoirs, Bruxelles, Bruylant, pp. 265-278.

Levy P., (1998), Le virtuel : étude d'un processus de transformation, Paris, La découverte, 159 p.

Mattelart T., (2009), TIC \& diaspora, TIC \& société, vol. 3, n¹-2, http://ticetsociete.revues.org/612 , date de la dernière visite : 25 décembre 2011.

Miere-Pélage Th., (2008), Usages de la téléphonie mobile en contexte de sous-développement, in Kiyindou A. (dir.), Communication pour le développement, Analyse critique des dispositifs et pratiques professionnelles au Congo, Bruxelles, EME, pp. 157-178.

Pires-Hester Laura J., (1999), The emergence of bilateral diaspora ethnicity among Cape VerdeanAmericans, in Okpewho I. et al., The African Diaspora : African Origins and New World Identities, Indiana University Press, pp. 485-503.

Scopsi C., (2004), Représentation des TIC et multiterritorialité : le cas des télé et cyber boutiques de Château-Rouge à Paris, in Cheneau-Loquay A., Technologies de la communication et mondialisation en Afrique, Paris, Karthala, pp. 275-293.

Zablocki O., (2001), Construire et habiter l'Internet, http://www.radiophare.net/RadioPhare, date de la dernière visite : 24 septembre 2010 .

\section{RÉSUMÉS}

Les membres de la diaspora noire se servent des Technologies de l'Information et de la Communication (TIC), téléphone mobile, messagerie électronique, sites Internet et blogs, etc. pour mieux se comprendre, discuter des problèmes de développement et participer de façon plus efficace à leur résolution. Les Africains de la diaspora cherchent, avant tout, à maintenir des contacts avec leurs amis et leurs parents restés « au Pays ». La reconstruction du lien social et la reconstruction identitaire apparaissent, dans ce cadre, comme sources de motivation des internautes.

Members of the African and Black diaspora use information and communication technologies (ICT), mobile phones, electronic mail, websites, and blogs to interact and discuss problems of development and participate in a more effective way in their resolution. Above all, Africans of the Diaspora try to maintain contact with friends and relatives back home. From this perspective, the reconstruction of social links and identity emerge as sources of motivation for Internet users. 
INDEX

Mots-clés : TIC, diaspora, identité, lien social, Afrique

Keywords : ICT, diaspora, identity, social ties

\section{AUTEURS}

\section{ALAIN KIYINDOU}

MICA - Université de Bordeaux 3

\section{THÉODORA MIÉRÉ PÉLAGE}

LAREQUOI - Université de Versailles Saint-Quentin-en-Yvelines 